\title{
Sharp Bounds of the Coefficient Results for the Family of Bounded Turning Functions Associated with a Petal-Shaped Domain
}

\author{
Omar M. Barukab, ${ }^{1}$ Muhammad Arif $\left(\mathbb{D}^{2},{ }^{2}\right.$ Muhammad Abbas, ${ }^{2}$ and Sher Afzal Khan ${ }^{2}$ \\ ${ }^{1}$ Faculty of Computing and Information Technology, King Abdulaziz University, P.O. Box 344, Rabigh, 21911 Jeddah, Saudi Arabia \\ ${ }^{2}$ Faculty of Physical and Numerical Sciences, Abdul Wali Khan University Mardan, Mardan 23200, Pakistan
}

Correspondence should be addressed to Muhammad Arif; marifmaths@awkum.edu.pk

Received 14 January 2021; Revised 27 January 2021; Accepted 6 February 2021; Published 20 February 2021

Academic Editor: Gangadharan Murugusundaramoorthy

Copyright (C) 2021 Omar M. Barukab et al. This is an open access article distributed under the Creative Commons Attribution License, which permits unrestricted use, distribution, and reproduction in any medium, provided the original work is properly cited.

The goal of this article is to determine sharp inequalities of certain coefficient-related problems for the functions of bounded turning class subordinated with a petal-shaped domain. These problems include the bounds of first three coefficients, the estimate of Fekete-Szegö inequality, and the bounds of second- and third-order Hankel determinants.

\section{Preliminary Concepts}

Let the family of holomorphic (or analytic) functions in the region (or domain) of unit disc $\mathbb{D}=\{z \in \mathbb{C}:|z|<1\}$ be described by the symbol $\mathscr{H}(\mathbb{D})$ and let $\mathscr{A}$ be the subfamily of $\mathscr{H}(\mathbb{D})$ which is defined by

$$
\mathscr{A}=\left\{f \in \mathscr{H}(\mathbb{D}): f(z)=\sum_{k=1}^{\infty} a_{k} z^{k}\left(\text { with } a_{1}=1\right)\right\} .
$$

Further, the set $\mathcal{S} \subset \mathscr{A}$ contains all normalized univalent functions in $\mathbb{D}$. For two functions $F_{1}, F_{2} \in \mathscr{H}(\mathbb{D})$, we say that $F_{1}$ is subordinate to $F_{2}$, written symbolically by $F_{1} \prec F_{2}$, if there exists a Schwarz function $v$ with $v(0)=0$ and $|v(z)|<1$ that is analytic in $\mathbb{D}$ such that $f(z)=g(v(z)), z \in \mathbb{D}$. However, if $F_{2}$ is univalent in $\mathbb{D}$, then the following relation holds:

$$
F_{1}(z) \prec F_{2}(z),(z \in \mathbb{D}) \Leftrightarrow F_{1}(0)=F_{2}(0) \text { and } F_{1}(\mathbb{D}) \subset F_{2}(\mathbb{D}) \text {. }
$$

In geometric function theory, the most basic and important subfamilies of the set $\mathcal{S}$ are the family $\mathcal{S}^{*}$ of starlike functions and the family $\mathscr{C}$ of convex functions which are defined as follows:

$$
\begin{gathered}
\mathscr{C}=\left\{f \in \mathscr{A}: \frac{\left(z f^{\prime}(z)\right)^{\prime}}{f^{\prime}(z)} \prec \Lambda(z)(z \in \mathbb{D})\right\}:=\mathscr{C}(\Lambda), \\
\mathcal{S}^{*}=\left\{f \in \mathscr{A}: \frac{z f^{\prime}(z)}{f(z)} \prec \Lambda(z)(z \in \mathbb{D})\right\}:=\mathcal{S}^{*}(\Lambda),
\end{gathered}
$$

with

$$
\Lambda(z)=1+2 \sum_{n=2}^{\infty} z^{n}:=\frac{1+z}{1-z},(z \in \mathbb{D}) .
$$

By varying the function $\Lambda(z)$ in (18), we get some subfamilies of the set $\mathcal{S}^{*}$ which have significant geometric sense. For example,

(i) If we take $\Lambda(z)=(1+M z) /(1+N z)$ with $-1 \leq N<$ $M \leq 1$, then the deduced family 


$$
\mathcal{S}^{*}[M, N] \equiv \mathcal{S}^{*}\left(\frac{1+M z}{1+N z}\right)
$$

is described by the functions of the Janowski starlike family established in [1] and later studied in different directions in $[2,3]$

(ii) The family $\mathcal{S}_{L}^{*} \equiv \mathcal{S}^{*}(\Lambda(z))$ with $\Lambda(z)=\sqrt{1+z}$ was developed in [4] by Sokól and Stankiewicz. The image of the function $\Lambda(z)=\sqrt{1+z}$ demonstrates that the image domain is bounded by the Bernoulli's lemniscate right-half plan specified by $\left|w^{2}-1\right|<1$

(iii) By selecting $\Lambda(z)=1+\sin z$, the class $\mathcal{S}^{*}(\Lambda(z))$ lead to the family $\mathcal{S}_{\sin }^{*}$ which was explored in [5] while $\mathcal{S}_{e}^{*} \equiv \mathcal{S}^{*}\left(e^{z}\right)$ has been produced in the article [6] and later studied in [7]

(iv) The family $\mathcal{S}_{c}^{*}:=\mathcal{S}^{*}(\Lambda(z))$ with $\Lambda(z)=1+(4 / 3) z$ $+(2 / 3) z^{2}$ was contributed by Sharma and his coauthors [8] which contains function $f \in \mathscr{A}$ such that $z$ $f^{\prime}(z) / f(z)$ is located in the region bounded by the cardioid given by

$$
\left(9 x^{2}+9 y^{2}-18 x+5\right)^{2}-16\left(9 x^{2}+9 y^{2}-6 x+1\right)=0
$$

(v) The family $\mathcal{S}_{R}^{*} \equiv \mathcal{S}^{*}(\Lambda(z))$ with $\Lambda(z)=1+(z /(\sqrt{ } 2$ $+1))((\sqrt{ } 2+1+z) /(\sqrt{ } 2+1-z))$ is studied in [9] while $\mathcal{S}_{\cos }^{*}:=\mathcal{S}^{*}(\cos (z))$ and $\mathcal{S}_{\cosh }^{*}:=\mathcal{S}^{*}(\cosh (z))$ were recently examined by Bano and Raza [10] and Alotaibi et al. [11], respectively

(vi) If we consider $\Lambda(z)=1 \sinh ^{-1} z$, then the class $\mathcal{S}_{\rho}^{*}:=\mathcal{S}^{*}\left(1+\sinh ^{-1} z\right)$ was provided by Kumar and Arora [12] and is defined as a function $f \in \mathscr{A}$ which is in the family $\mathcal{S}_{\rho}^{*}$ if (18) holds for the function $\Lambda(z)=\rho(z)$, where

$$
\rho(z)=1+\sinh ^{-1} z
$$

Clearly, the function $\rho(z)$ is a multivalued function and has the branch cuts about the line segments $(-i \infty,-i) \cup(i, i \infty)$, on the imaginary axis, and hence, it is holomorphic in $\mathbb{D}$. In a geometric point of view, the function $\rho(z)$ maps the unit disc $\mathbb{D}$ onto a petal-shaped region $\Omega_{\rho}$,

$$
\Omega_{\rho}=\{w \in \mathbb{C}:|\sinh (w-1)|<1\} .
$$

Using this idea, we now consider a subfamily $\mathscr{B} \mathscr{T}_{s}$ of analytic functions as

$$
\mathscr{B} \mathscr{T}_{s}=\left\{f \in \mathscr{A}: f^{\prime}(z) \prec \tilde{\Lambda}(z) \text {, and } \tilde{\Lambda}(z) \text { is given by }(8)\right\} \text {. }
$$

If we take the function $\Lambda(z)$, given by (4), instead of $\tilde{\Lambda}(z)$ in (9), we get the familiar class $\mathscr{R}$ of bounded turning functions. From the statement of the Nashiro-Warschowski theorem, it follows that the functions in $\mathscr{R}$ are univalent in $\mathbb{D}$. The properties of this class was studied extensively by the researchers, see [13-16].

The Hankel determinant $\mathscr{H} \mathscr{D}_{q, n}(f)$ (with $q, n \in \mathbb{N}=\{1,2$, $\cdots\}$ and $a_{1}=1$ ) for a function $f \in \mathcal{S}$ of the series form (1) was given by Pommerenke $[17,18]$ as

$$
\mathscr{H} \mathscr{D}_{q, n}(f):=\left|\begin{array}{cccc}
a_{n} & a_{n+1} & \cdots & a_{n+q-1} \\
a_{n+1} & a_{n+2} & \cdots & a_{n+q} \\
\vdots & \vdots & \cdots & \vdots \\
a_{n+q-1} & a_{n+q} & \cdots & a_{n+2 q-2}
\end{array}\right| .
$$

Specifically, the first-, second-, and third-order Hankel determinants, respectively, are

$$
\begin{aligned}
\mathscr{H} \mathscr{D}_{2,1}(f) & =\left|\begin{array}{cc}
1 & a_{2} \\
a_{2} & a_{3}
\end{array}\right|=a_{3}-a_{2}^{2}, \\
\mathscr{H} \mathscr{D}_{2,2}(f) & =\left|\begin{array}{ll}
a_{2} & a_{3} \\
a_{3} & a_{4}
\end{array}\right|=a_{2} a_{4}-a_{3}^{2}, \\
\mathscr{H} \mathscr{D}_{3,1}(f) & =\left|\begin{array}{ccc}
1 & a_{2} & a_{3} \\
a_{2} & a_{3} & a_{4} \\
a_{3} & a_{4} & a_{5}
\end{array}\right| \\
& =a_{3}\left(a_{2} a_{4}-a_{3}^{2}\right)-a_{4}\left(a_{4}-a_{2} a_{3}\right)+a_{5}\left(a_{3}-a_{2}^{2}\right) .
\end{aligned}
$$

In literature, there are relatively few findings in relation to the Hankel determinant for the function $f$ belongs to the general family $\mathcal{S}$. For the function $f \in \mathcal{S}$, the best established sharp inequality is $\left|\mathscr{H} \mathscr{D}_{2, n}(f)\right| \leq \lambda \sqrt{n}$, where $\lambda$ is absolute constant, which is due to Hayman [19]. Further, for the same class $\mathcal{S}$, it was obtained in [20] that

$$
\begin{aligned}
& \left|\mathscr{H} \mathscr{D}_{2,2}(f)\right| \leq \lambda \text {, for } 1 \leq \lambda \leq \frac{11}{3}, \\
& \left|\mathscr{H} \mathscr{D}_{3,1}(f)\right| \leq \mu \text {, for } \frac{4}{9} \leq \mu \leq \frac{32+\sqrt{285}}{15} .
\end{aligned}
$$

The growth of $\left|\mathscr{H} \mathscr{D}_{q, n}(f)\right|$ has often been evaluated for different subfamilies of the set $\mathcal{S}$ of univalent functions. For example, the sharp bound of $\left|\mathscr{H} \mathscr{D}_{2,2}(f)\right|$, for the subfamilies $\mathscr{C}, \mathcal{S}^{*}$, and $\mathscr{R}$ of the set $\mathcal{S}$, was measured by Janteng et al. [21, 22]. These bounds are 


$$
\left|\mathscr{H}_{2,2}(f)\right| \leq \begin{cases}\frac{1}{8}, & \text { for } f \in \mathscr{C}, \\ 1, & \text { for } f \in \mathcal{S}^{*} \\ \frac{4}{9}, & \text { for } f \in \mathscr{R}\end{cases}
$$

The exact bound for the collection of close-to-convex functions of such a specific determinant is still unavailable (see [23]). On the other hand, for the set of Bazilevic functions, the best estimate of $\left|\mathscr{H} \mathscr{D}_{2,2}(f)\right|$ was proved by Krishna and RamReddy [24]. For more work on $\left|\mathscr{H} \mathscr{D}_{2,2}(f)\right|$, see References [25-29].

It is very obvious from the formulae provided in (11) that the estimate of $\left|\mathscr{H} \mathscr{D}_{3,1}(f)\right|$ is far more complicated compared with finding the bound of $\left|\mathscr{H} \mathscr{D}_{2,2}(f)\right|$. In the first paper on $\left|\mathscr{H} \mathscr{D}_{3,1}(f)\right|$, published in 2010, Babalola [30] obtained the upper bound of $\left|\mathscr{H} \mathscr{D}_{3,1}(f)\right|$ for the families of $\mathscr{C}, \mathcal{S}^{*}$, and $\mathscr{R}$. He obtained the following bounds:

$$
\left|\mathscr{C D}_{3,1}(f)\right| \leq \begin{cases}0.714 \cdots, & \text { for } f \in \mathscr{C}, \\ 16, & \text { for } f \in \mathcal{S}^{*}, \\ 0.742 \cdots, & \text { for } f \in \mathscr{R} .\end{cases}
$$

Later on, using the same methodology, some other authors [31-35] published their work concerning $\left|\mathscr{H} \mathscr{D}_{3,1}(f)\right|$ for different subfamilies of analytic and univalent functions. In 2017, Zaprawa [36] improved Babalola's [30] results by applying a new technique which is given as

$$
\left|\mathscr{H} \mathscr{D}_{3,1}(f)\right| \leq \begin{cases}\frac{49}{540}, & \text { for } f \in \mathscr{C}, \\ 1, & \text { for } f \in \mathcal{S}^{*}, \\ \frac{41}{60}, & \text { for } f \in \mathscr{R} .\end{cases}
$$

He argues that such limits are indeed not the best ones. After that, in 2018, Kwon et al. [37] enhanced Zaprawa's bound for $f \in \mathcal{S}^{*}$ and showed that $\left|\mathscr{H} \mathscr{D}_{3,1}(f)\right| \leq 8 / 9$, but it is still not the best possible. The firstly examined papers in which the authors obtained the sharp bounds of $\left|\mathscr{H}_{3,1}(f)\right|$ came to the reader's hands in 2018. Such papers have been written by Kowalczyk et al. [38] and Lecko et al. [39]. These results are given as

$$
\left|\mathscr{H} \mathscr{D}_{3,1}(f)\right| \leq \begin{cases}\frac{4}{135}, & \text { for } f \in \mathscr{C}, \\ \frac{1}{9}, & \text { for } f \in \mathcal{S}^{*}\left(\frac{1}{2}\right),\end{cases}
$$

where $\mathcal{S}^{*}(1 / 2)$ indicates the starlike function family of order $1 / 2$. We would also like to acknowledge the research provided by Mahmood et al. [40] in which they examined the third Hankel determinant in the $q$-analog for a subfamily of starlike functions and for more contribution of such type families, see $[41,42]$. In the present article, our aim is to calculate the sharp bounds of some of the problems related to Hankel determi- nant for the class $\mathscr{B} \mathscr{T}_{s}$ of bounded turning functions connected with a petal-shaped domain.

\section{A Set of Lemmas}

Definition 1. Let $\mathscr{P}$ represent the class of all functions $p$ that are holomorphic in $\mathbb{D}$ with $\mathfrak{R e}(p(z))>0$ and has the series representation

$$
p(z)=1+\sum_{n=1}^{\infty} c_{n} z^{n}(z \in \mathbb{D}) .
$$

For the proofs of our key findings, we need the following lemma. It contains the well-known formula for $c_{2}$, see [43], the formula for $c_{3}$ due to Libera and Zlotkiewicz [44], and the formula for $c_{4}$ proved in [45].

Lemma 2. Let $p \in \mathscr{P}$ has the series form ((17)). Then, for $x$, $\sigma, \rho \in \overline{\mathbb{D}}=\mathbb{D} \cup\{1\}$,

$2 c_{2}=c_{1}^{2}+x\left(4-c_{1}^{2}\right)$

$4 c_{3}=c_{1}^{3}+2\left(4-c_{1}^{2}\right) c_{1} x-c_{1}\left(4-c_{1}^{2}\right) x^{2}+2\left(4-c_{1}^{2}\right)\left(1-|x|^{2}\right) \sigma$,

$$
\begin{aligned}
8 c_{4}= & c_{1}^{4}+\left(4-c_{1}^{2}\right) x\left[c_{1}^{2}\left(x^{2}-3 x+3\right)+4 x\right]-4\left(4-c_{1}^{2}\right) \\
& \cdot\left(1-|x|^{2}\right)\left[c(x-1) z+\bar{x} \sigma^{2}-\left(1-|\sigma|^{2}\right) \rho\right] .
\end{aligned}
$$

Lemma 3. If $p \in \mathscr{P}$ and has the series form ((17)), then

$$
\begin{gathered}
\left|c_{n+k}-\mu c_{n} c_{k}\right| \leq 2 \max (1,|2 \mu-1|), \\
\left|c_{n}\right| \leq 2 \text { for } n \geq 1, \\
\left|J c_{1}^{3}-K c_{1} c_{2}+L c_{3}\right| \leq 2|J|+|K-2 J|+2|J-K+L|,
\end{gathered}
$$

with $J, K, L, \mu \in \mathbb{C}$ and for $B \in[0,1]$ with $B(2 B-1) \leq D \leq B$, we have

$$
\left|c_{3}-2 B c_{1} c_{2}+D c_{1}^{3}\right| \leq 2
$$

The inequalities (21), (22), (23), and (24) in the above lemma are taken from $[43,46],[47-49]$, and [50], respectively.

\section{Coefficient Inequalities for the Class $\mathscr{B} \mathscr{T}_{s}$}

We begin this section by finding the absolute values of the first three initial coefficients for the function of class $\mathscr{B} \mathscr{T}_{s}$. 
Theorem 4. If $f \in \mathscr{B} \mathscr{T}_{s}$ and has the series representation ((1)), then

$$
\begin{aligned}
& \left|a_{2}\right| \leq \frac{1}{2} \\
& \left|a_{3}\right| \leq \frac{1}{3} \\
& \left|a_{4}\right| \leq \frac{1}{4}
\end{aligned}
$$

These bounds are sharp.

Proof. Let $f \in \mathscr{B} \mathscr{T}_{s}$. Then, (9) can be written in the form of the Schwarz function as

$$
f^{\prime}(z)=1+\sinh ^{-1}(w(z)),(z \in \mathbb{D}) .
$$

Now, if $p \in \mathscr{P}$, then it may be written in terms of the Schwarz function $w$ by

$$
p(z)=\frac{1+w(z)}{1-w(z)}=1+c_{1} z+c_{2} z^{2}+c_{3} z^{3}+\cdots,
$$

equivalently,

$$
w(z)=\frac{p(z)-1}{p(z)+1}=\frac{c_{1} z+c_{2} z^{2}+c_{3} z^{3}+c_{4} z^{4}+\cdots}{2+c_{1} z+c_{2} z^{2}+c_{3} z^{3}+c_{4} z^{4}+\cdots} .
$$

From (1), we easily get

$$
f^{\prime}(z)=1+2 a_{2} z+3 a_{3} z^{2}+4 a_{4} z^{3}+5 a_{5} z^{4}+\cdots
$$

By simplification and using the series expansion (28), we obtain

$$
\begin{aligned}
1+\sinh ^{-1}(w(z))= & +\left(\frac{1}{2} c_{1}\right) z+\left(\frac{1}{2} c_{2}-\frac{1}{4} c_{1}^{2}\right) z^{2} \\
& +\left(\frac{1}{2} c_{3}+\frac{5}{48} c_{1}^{3}-\frac{1}{2} c_{1} c_{2}\right) z^{3} \\
& \cdot\left(\frac{1}{2} c_{4}-\frac{1}{4} c_{2}^{2}-\frac{1}{32} c_{1}^{4}+\frac{5}{16} c_{1}^{2} c_{2}-\frac{1}{2} c_{1} c_{3}\right) z^{4}+\cdots
\end{aligned}
$$

Comparing (29) and (30),we get

$$
\begin{aligned}
& a_{2}=\frac{1}{4} c_{1}, \\
& a_{3}=\frac{1}{3}\left(\frac{1}{2} c_{2}-\frac{1}{4} c_{1}^{2}\right), \\
& a_{4}=\frac{1}{4}\left(\frac{1}{2} c_{3}+\frac{5}{48} c_{1}^{3}-\frac{1}{2} c_{1} c_{2}\right), \\
& a_{5}=\frac{1}{5}\left(\frac{1}{2} c_{4}-\frac{1}{4} c_{2}^{2}-\frac{1}{32} c_{1}^{4}+\frac{5}{16} c_{1}^{2} c_{2}-\frac{1}{2} c_{1} c_{3}\right) .
\end{aligned}
$$

For $a_{2}$, implementing (22) in (31), we obtain

$$
\left|a_{2}\right| \leq \frac{1}{2}
$$

For $a_{3}$, reordering (32), we get

$$
a_{3}=\frac{1}{6}\left(c_{2}-\frac{1}{2} c_{1} c_{1}\right)
$$

and using (21), we have

$$
\left|a_{3}\right| \leq \frac{1}{3}
$$

For $a_{4}$, we can rewrite (33) as

$$
\left|a_{4}\right|=\frac{1}{4}\left|\frac{5}{48} c_{1}^{3}-\frac{1}{2} c_{1} c_{2}+\frac{1}{2} c_{3}\right|
$$

Application of triangle inequality plus (23), we get

$$
\left|a_{4}\right| \leq \frac{1}{4}\left[2\left|\frac{5}{48}\right|+2\left|\frac{1}{2}-2\left(\frac{5}{48}\right)\right|+2\left|\frac{5}{48}-\frac{1}{2}+\frac{1}{2}\right|\right] .
$$

By simple calculations, we obtain

$$
\left|a_{4}\right| \leq \frac{1}{4}
$$

These outcomes are sharp. For this, we consider a function

$$
f_{n}^{\prime}(z)=1+\sinh ^{-1}\left(z^{n}\right), \text { for } n=1,2,3 .
$$

Thus, we have

$$
\begin{aligned}
& f_{1}(z)=\int_{0}^{z}\left(1+\sinh ^{-1}(t)\right) d t=z+\frac{1}{2} z^{2}-\frac{1}{24} z^{4}+\cdots \\
& f_{2}(z)=\int_{0}^{z}\left(1+\sinh ^{-1}\left(t^{2}\right)\right) d t=z+\frac{1}{3} z^{3}-\frac{1}{42} z^{7}+\cdots \\
& f_{3}(z)=\int_{0}^{z}\left(1+\sinh ^{-1}\left(t^{3}\right)\right) d t=z+\frac{1}{4} z^{4}-\frac{1}{60} z^{10}+\cdots
\end{aligned}
$$

Now, we discussed about the Hankel determinant problem, which is explicitly related to the Fekete-Szegö functional which is an extraordinary instance of the Hankel determinant.

Theorem 5. If $f$ of the form ((1)) belongs to $\mathscr{B} \mathscr{T}_{s}$, then

$$
\left|a_{3}-\gamma a_{2}^{2}\right| \leq \max \left\{1, \frac{3|\gamma|}{4}\right\}, \text { for } \gamma \in \mathbb{C}
$$

This inequality is sharp. 
Proof. Employing (31) and (32), we may write

$$
\left|a_{3}-\gamma a_{2}^{2}\right|=\left|\frac{c_{2}}{6}-\frac{c_{1}^{2}}{12}-\gamma \frac{c_{1}^{2}}{16}\right|
$$

By rearranging, it yields

$$
\left|a_{3}-\gamma a_{2}^{2}\right|=\frac{1}{6}\left|\left(c_{2}-\left(\frac{3 \gamma+4}{8}\right) c_{2}\right)\right|
$$

Application of (21) leads us to

$$
\left|a_{3}-\gamma a_{2}^{2}\right| \leq \frac{1}{6} \max \left\{2,2\left|2 \frac{3 \gamma+4}{8}-1\right|\right\} \text {. }
$$

After the simplification, we obtain

$$
\left|a_{3}-\gamma a_{2}^{2}\right| \leq \frac{1}{3} \max \left\{1, \frac{3|\gamma|}{4}\right\}
$$

The required result is sharp and is determined by

$$
f_{2}(z)=\int_{0}^{z}\left(1+\sinh ^{-1}\left(t^{2}\right)\right) d t=z+\frac{1}{3} z^{3}-\frac{1}{42} z^{7}+\cdots
$$

Theorem 6. If $f$ has the form ((1)) belongs to $\mathscr{B} \mathscr{T}_{s}$, then

$$
\left|a_{2} a_{3}-a_{4}\right| \leq \frac{1}{4}
$$

This inequality is sharp.

Proof. Using (31), (32), and (33), we have

$$
\left|a_{2} a_{3}-a_{4}\right|=\frac{1}{8}\left|c_{3}-2\left(\frac{2}{3}\right) c_{1} c_{2}+\frac{7}{24} c_{1}^{3}\right| .
$$

From (24), we have

$$
0 \leq B=\frac{2}{3} \leq 1, B=\frac{2}{3} \geq D=\frac{7}{24}
$$

and also satisfy

$$
B(2 B-1)=\frac{2}{3}\left(\frac{4}{3}-1\right) \leq D=\frac{7}{24}
$$

Thus, by using (24), we have

$$
\left|a_{2} a_{3}-a_{4}\right| \leq \frac{1}{4}
$$

Equality is achieved by using

$$
f_{3}(z)=\int_{0}^{z}\left(1+\sinh ^{-1}\left(t^{3}\right)\right) d t=z+\frac{1}{4} z^{4}-\frac{1}{60} z^{10}+\cdots
$$

Next, we will determine the second-order Hankel determinant $\mathscr{H} \mathscr{D}_{2,2}(f)$ for $f \in \mathscr{B} \mathscr{T}_{s}$.

Theorem 7. If $f$ belongs to $\mathscr{B} \mathscr{T}_{s}$, then the second Hankel determinant

$$
\left|\mathscr{H} \mathscr{D}_{2,2}(f)\right|=\left|a_{2} a_{4}-a_{3}^{2}\right| \leq \frac{1}{9} .
$$

This result is the best possible.

Proof. From (31), (32), and (33), we have

$$
\mathscr{H} \mathscr{D}_{2,2}(f)=-\frac{1}{2304} c_{1}^{4}-\frac{1}{288} c_{1}^{2} c_{2}+\frac{1}{32} c_{1} c_{3}-\frac{1}{36} c_{2}^{2} .
$$

Using (18) and (19) to express $c_{2}$ and $c_{3}$ in terms of $c_{1}$ and noting that without loss in generality we can write $c_{1}=c$, with $0 \leq c \leq 2$, we obtain

$$
\begin{aligned}
\left|\mathscr{H} \mathscr{D}_{2,2}(f)\right|= & \mid-\frac{1}{768} c^{4}-\frac{1}{128} c^{2}\left(4-c^{2}\right) x^{2}-\frac{1}{144}\left(4-c^{2}\right)^{2} x^{2} \\
& +\frac{1}{64} c\left(4-c^{2}\right)\left(1-|x|^{2}\right) z \mid
\end{aligned}
$$

with the aid of the triangle inequality and replacing $|z| \leq 1$, $x \mid=b$, with $b \leq 1$. So,

$$
\begin{aligned}
\left|\mathscr{H} \mathscr{D}_{2,2}(f)\right| & \leq \frac{1}{768} c^{4}+\frac{1}{128} c^{2}\left(4-c^{2}\right) b^{2}+\frac{1}{144}\left(4-c^{2}\right)^{2} b^{2} \\
+\frac{1}{64} b\left(4-c^{2}\right)\left(1-b^{2}\right): & =\phi(c, b) .
\end{aligned}
$$

It is a simple exercise to show that $\phi^{\prime}(c, b) \geq 0$ on $[0,1]$, so that $\phi(c, b) \leq \phi(c, 1)$. Putting $b=1$ gives

$$
\left|\mathscr{H} \mathscr{D}_{2,2}(f)\right| \leq \frac{1}{768} c^{4}+\frac{1}{128} c^{2}\left(4-c^{2}\right)+\frac{1}{144}\left(4-c^{2}\right)^{2}:=\phi(c, 1) .
$$

Also, $\phi^{\prime}(c, 1)<0$, and so $\phi(c, 1)$ is a decreasing function. Thus, the maximum value at $c=0$ is

$$
\left|\mathscr{H} \mathscr{D}_{2,2}(f)\right| \leq \frac{16}{144}=\frac{1}{9} .
$$

The required second Hankel determinant is sharp and is obtained by

$$
f_{2}(z)=\int_{0}^{z}\left(1+\sinh ^{-1}\left(t^{2}\right)\right) d t=z+\frac{1}{3} z^{3}-\frac{1}{42} z^{7}+\cdots
$$

\section{Third-Order Hankel Determinant}

We will now determine the third-order Hankel determinant $\mathscr{H} \mathscr{D}_{3,1}(f)$ for $f \in \mathscr{B} \mathscr{T}_{s}$. 
Theorem 8. If $f$ belongs to $\mathscr{B} \mathscr{T}_{s}$, then the third Hankel determinant

$$
\left|\mathscr{H} \mathscr{D}_{3,1}(f)\right| \leq \frac{1}{16} \text {. }
$$

This result is sharp.

Proof. The third Hankel determinant can be written as

$\mathscr{H} \mathscr{D}_{3,1}(f)=\left(a_{2} a_{4}-a_{3}^{2}\right) a_{3}-\left(a_{1} a_{4}-a_{2} a_{3}\right) a_{4}+\left(a_{1} a_{3}-a_{2}^{2}\right) a_{5}$.

After simplification of the above equation, we have

$$
\mathscr{H} \mathscr{D}_{3,1}(f)=2 a_{2} a_{3} a_{4}-a_{3}^{3}-a_{4}^{2}+a_{3} a_{5}-a_{2}^{2} a_{5} .
$$

Let $c_{1}=c$ and putting the estimations of $a_{i}$ 's from (31), (32), (33), and (34), we get

$$
\begin{aligned}
\mathscr{H} \mathscr{D}_{3,1}(f)= & \frac{1}{552960}\left(-151 c^{6}+144 c^{4} c_{2}+1584 c^{3} c_{3}-768 c^{2} c_{2}^{2}\right. \\
& \left.-8064 c^{2} c_{4}+13824 c c_{2} c_{3}-7168 c_{2}^{3}+9216 c_{2} c_{4}-8640 c_{3}^{2}\right) .
\end{aligned}
$$

To simplify computation, let $t=4-c^{2}$ in (18), (19), and (20). Now, using the simplified form of these formulae, we obtain

$$
\begin{array}{rl}
144 c^{4} c_{2}= & 72\left(c^{6}+c^{4} t x\right), \\
1584 c^{3} c_{3}= & 396 c^{6}+792 c^{4} t x-396 c^{4} t x^{2}+792 c^{3} t\left(1-|x|^{2}\right) \sigma, \\
768 c^{2} c_{2}^{2}= & 192 c^{6}+384 c^{4} t x+192 c^{2} t^{2} x^{2}, \\
8064 c^{2} c_{4}= & 1008 c^{4} t x^{3}-4032 c^{3} t x\left(1-|x|^{2}\right) \sigma \\
& -4032 c^{2} t \bar{x}\left(1-|x|^{2}\right) \sigma^{2}-3024 c^{4} t x^{2} \\
& +4032 c^{2} t\left(1-|x|^{2}\right)\left(1-|\sigma|^{2}\right) \rho+4032 c^{3} t\left(1-|x|^{2}\right) \sigma \\
& +3024 c^{4} t x+1008 c^{6}+4032 c^{2} t x^{2}, \\
& -1728 c^{2} t^{2} x^{3}-1728 c^{4} t x^{2}+3456 c t^{2} x\left(1-|x|^{2}\right) \sigma \\
& +3456 c^{2} t^{2} x^{2}+3456 c^{3} t\left(1-|x|^{2}\right) \sigma+5184 c^{4} t x \\
& +1728 c^{6}, \\
13824 c c_{2} c_{3} & 896 t^{3} x^{3}+2688 c^{2} t^{2} x^{2}+2688 c^{4} t x+896 c^{6}, \\
7168 c_{2}^{3}= & 2304 c^{2} t x^{2}+2304 t^{2} x^{3}+576 c^{6}+2304 c^{4} t x \\
& +2304 c^{3} t\left(1-|x|^{2}\right) \sigma+2304 c^{2} t\left(1-|x|^{2}\right)\left(1-|\sigma|^{2}\right) \rho \\
& +1728 c^{2} t^{2} x^{2}+2304 c t^{2} x\left(1-|x|^{2}\right) \sigma \\
& +2304 t^{2} x\left(1-|x|^{2}\right)\left(1-|\sigma|^{2}\right) \rho-1728 c^{4} t x^{2} \\
& -2304 c^{2} t \bar{x}\left(1-|x|^{2}\right) \sigma^{2}-2304 c^{3} t x\left(1-|x|^{2}\right) \sigma \\
& -1728 c^{2} t^{2} x^{3}-2304 t^{2} x \bar{x}\left(1-|x|^{2}\right) \sigma^{2}+576 c^{4} t x^{3} \\
& +576 c^{2} t^{2} x^{4}-2304 c t^{2} x^{2}\left(1-|x|^{2}\right) \sigma, \\
= &
\end{array}
$$

$$
\begin{aligned}
8640 c_{3}^{2}= & 540 c^{2} t^{2} x^{4}-2160 c t^{2} x^{2}\left(1-|x|^{2}\right) \sigma-2160 c^{2} t^{2} x^{3} \\
& -1080 c^{4} t x^{2}+2160 t^{2}\left(1-|x|^{2}\right)^{2} \sigma^{2} \\
& +4320 c t^{2} x\left(1-|x|^{2}\right) \sigma+2160 c^{2} t^{2} x^{2}+2160 c^{3} t\left(1-|x|^{2}\right) \sigma \\
& +2160 c^{4} t x+540 c^{6} .
\end{aligned}
$$

Substituting these expressions in (65), by simple but too long computation,

$$
\begin{aligned}
\mathscr{H} \mathscr{D}_{3,1}(f)= & \frac{1}{552960}\left\{-15 c^{6}+2304 t^{2} x^{3}-896 t^{3} x^{3}-1728 c^{2} t x^{2}\right. \\
& -432 c^{4} t x^{3}+252 c^{4} t x^{2}+96 c^{4} t x+36 c^{2} t^{2} x^{4} \\
& -1296 c^{2} t^{2} x^{3}+144 c^{2} t^{2} x^{2}-2160 t^{2}\left(1-|x|^{2}\right)^{2} \sigma^{2} \\
& +360 c^{3} t\left(1-|x|^{2}\right) \sigma+1728 c^{3} t x\left(1-|x|^{2}\right) \sigma \\
& +1728 c^{2} t \bar{x}\left(1-|x|^{2}\right) \sigma^{2}-1728 c^{2} t\left(1-|x|^{2}\right)\left(1-|\sigma|^{2}\right) \rho \\
& -144 c t^{2} x^{2}\left(1-|x|^{2}\right) \sigma-2304 t^{2} x \bar{x}\left(1-|x|^{2}\right) \sigma^{2}+1440 c t^{2} x\left(1-|x|^{2}\right) \sigma \\
& \left.+2304 t^{2} x\left(1-|x|^{2}\right)\left(1-|\sigma|^{2}\right) \rho\right\} .
\end{aligned}
$$

Since $t=4-c^{2}$,

$\mathscr{H} \mathscr{D}_{3,1}(f)=\frac{1}{552960}\left(v_{1}(c, x)+v_{2}(c, x) \sigma+v_{3}(c, x) \sigma^{2}+\Psi(c, x, \sigma) \rho\right)$,

where $\rho, x, \sigma \in \overline{\mathbb{D}}$, and

$$
\begin{aligned}
v_{1}(c, x)= & -15 c^{6}+\left(4-c^{2}\right)\left[( 4 - c ^ { 2 } ) \left(-1280 x^{3}-400 c^{2} x^{3}+36 c^{2} x^{4}\right.\right. \\
& \left.\left.+144 c^{2} x^{2}\right)-1728 c^{2} x^{2}-432 c^{4} x^{3}+252 c^{4} x^{2}+96 c^{4} x\right], \\
v_{2}(c, x)= & \left(4-c^{2}\right)\left(1-|x|^{2}\right)\left[\left(4-c^{2}\right)\left(1440 c x-144 c x^{2}\right)\right. \\
& \left.+1728 c^{3} x+360 c^{3}\right], \\
v_{3}(c, x)= & \left(4-c^{2}\right)\left(1-|x|^{2}\right)\left[\left(4-c^{2}\right)\left(-144 x^{2}-2160\right)+1728 c^{2} \bar{x}\right], \\
\Psi(c, x, z)= & \left(4-c^{2}\right)\left(1-|x|^{2}\right)\left(1-|\sigma|^{2}\right)\left[-1728 c^{2}+2304 x\left(4-c^{2}\right)\right] .
\end{aligned}
$$

Now, by using $|x|=x,|\sigma|=y$ and utilizing the fact $|\rho| \leq 1$, we get

$$
\begin{gathered}
\left|\mathscr{H} \mathscr{D}_{3,1}(f)\right| \leq \frac{1}{552960}\left(\left|v_{1}(c, x)\right|+\left|v_{2}(c, x)\right| y+\left|v_{3}(c, x)\right| y^{2}\right. \\
+|\Psi(c, x, \sigma)|) \\
\leq \frac{1}{552960} G(c, x, y)
\end{gathered}
$$

where

$G(c, x, y)=g_{1}(c, x)+g_{2}(c, x) y+g_{3}(c, x) y^{2}+g_{4}(c, x)\left(1-y^{2}\right)$, 
with

$$
\begin{aligned}
g_{1}(c, x)= & 15 c^{6}+\left(4-c^{2}\right)\left[( 4 - c ^ { 2 } ) \left(1280 x^{3}+400 c^{2} x^{3}+36 c^{2} x^{4}\right.\right. \\
& \left.\left.+144 c^{2} x^{2}\right)+1728 c^{2} x^{2}+432 c^{4} x^{3}+252 c^{4} x^{2}+96 c^{4} x\right], \\
g_{2}(c, x)= & \left(4-c^{2}\right)\left(1-x^{2}\right)\left[\left(4-c^{2}\right)\left(1440 c x+144 c x^{2}\right)+1728 c^{3} x+360 c^{3}\right], \\
g_{3}(c, x)= & \left(4-c^{2}\right)\left(1-x^{2}\right)\left[\left(4-c^{2}\right)\left(144 x^{2}+2160\right)+1728 c^{2} \bar{x}\right], \\
g_{4}(c, x)= & \left(4-c^{2}\right)\left(1-x^{2}\right)\left[1728 c^{2}+2304 x\left(4-c^{2}\right)\right] .
\end{aligned}
$$

Clearly, in the last four functions, $g_{2}(c, x)$ and $g_{3}(c, x)$ are nonnegative in the interval $[0,2] \times[0,1]$. So from (70) along with $y=|\sigma|$ in the interval $[0,1]$, we get

$$
G(c, x, y)=G(c, x, 1) .
$$

Therefore,

$$
G(c, x, 1)=g_{1}(c, x)+g_{2}(c, x)+g_{3}(c, x)+g_{4}(c, x)=F(c, x) \text {. }
$$

Here, we shall maximize $F(c, x)$ over the interval $[0,2] \times$ $[0,1]$. For this purpose, we consider possible cases:

(i) By taking $x=0$, we have

$$
F(c, 0)=15 c^{6}-360 c^{5}+2160 c^{4}+144 c^{3}-17280 c^{2}+34560=f_{1}(c) .
$$

Since $f_{1}^{\prime}(c)<0$ in $[0,2]$, so, $f_{1}(c)$ is decreasing over $[0,2]$. Thus, $f_{1}(c)$ has its maxima at $c=0$ which is equal to 34560

(ii) By taking $x=1$, we have

$$
F(c, 1)=-185 c^{6}-1968 c^{4}+5952 c^{2}+20480=f_{2}(c) .
$$

As $f_{2}^{\prime}(c)=0$ has its optimum point at $c_{0}=0.674788$. Therefore, $f_{2}(c)$ is an increasing function for $c \leq c_{0}$ and decreasing for $c_{0} \leq c$. Hence, $f_{2}(c)$ has its maxima at $c=c_{0}$ that is approximately equal to 22764.68167

(iii) By taking $c=0$, we have

$$
F(0, x)=-2304 x^{4}+20480 x^{3}-32256 x^{2}+34560=f_{3}(x) .
$$

As $f_{3}^{\prime}(x)<0$ over $[0,1]$, so, $f_{3}(x)$ is decreasing over $[0,1]$.
Thus, $f_{3}(x)$ has its maxima at $x=0$ which is equal to 34560 . Now, by taking $c=2$, we obtain

$$
F(2, x) \leq 960
$$

(iv) When $(c, x)$ lies in $[0,2] \times[0,1]$. Then, some simple computation shows that there exists real solution for these equations

$$
\begin{aligned}
& \frac{\partial F(c, x)}{\partial x}=0, \\
& \frac{\partial F(c, x)}{\partial c}=0,
\end{aligned}
$$

lies inside this region $[0,2] \times[0,1]$ at $(c, x) \approx(0,0)$. Consequently, we obtain

$$
F(c, x)=34560 .
$$

Thus, from all the above cases, we conclude that

$$
G(c, x, y) \leq 34560 \text { on }[0,2] \times[0,1] \times[0,1] .
$$

From (71), we can write

$$
\left|\mathscr{H} \mathscr{D}_{3,1}(f)\right| \leq \frac{1}{552960} G(c, x, y) \leq \frac{1}{16} \approx 0.0625 .
$$
function

If $f \in \mathscr{B T}_{s}$, then the equality is obtained from the

$$
f_{3}(z)=\int_{0}^{z}\left(1+\sinh ^{-1}\left(t^{3}\right)\right) d t=z+\frac{1}{4} z^{4}-\frac{1}{60} z^{10}+\cdots .
$$

\section{Conclusion}

For the family of bounded turning functions connected with a petal-shaped domain, we studied the problems such as the bounds of the first three coefficients, the estimate of the Fekete-Szegö inequality, and the bounds of Hankel determinants of order three. All the bounds which we investigated are sharp.

\section{Data Availability}

We have not used any data.

\section{Conflicts of Interest}

The authors declare no conflict of interest.

\section{Authors' Contributions}

All authors contributed equally in this research paper. 


\section{Acknowledgments}

This project was funded by the Deanship of Scientific Research (DSR), King Abdulaziz University, under grant No. DF-762-830-1441. Therefore, the authors gratefully acknowledge the DSR technical and financial support.

\section{References}

[1] W. Janowski, "Extremal problems for a family of functions with positive real part and for some related families," Annales Polonici Mathematici, vol. 23, no. 2, pp. 159-177, 1970.

[2] M. Arif, K. Ahmad, J.-L. Liu, and J. Sokół, "A new class of analytic functions associated with Sălăgean operator," Journal of Function Spaces, vol. 2019, Article ID 6157394, 8 pages, 2019.

[3] K. I. Noor and M. Arif, "Mapping properties of an integral operator," Applied Mathematics Letters, vol. 25, no. 11, pp. 1826-1829, 2012.

[4] J. Sokół and J. Stankiewicz, "Radius of convexity of some subclasses of strongly starlike functions," Zeszyty Naukowe Politechniki Rzeszowskiej, vol. 19, pp. 101-105, 1996.

[5] N. E. Cho, V. Kumar, S. S. Kumar, and V. Ravichandran, "Radius problems for starlike functions associated with the sine function," Bulletin of the Iranian Mathematical Society, vol. 45, no. 1, pp. 213-232, 2019.

[6] R. Mendiratta, S. Nagpal, and V. Ravichandran, "On a subclass of strongly starlike functions associated with exponential function," Bulletin of the Malaysian Mathematical Sciences Society, vol. 38, no. 1, pp. 365-386, 2015.

[7] L. Shi, H. M. Srivastava, M. Arif, S. Hussain, and H. Khan, "An investigation of the third Hankel determinant problem for certain subfamilies of univalent functions involving the exponential function," Symmetry, vol. 11, p. 598, 2019.

[8] K. Sharma, N. K. Jain, and V. Ravichandran, "Starlike functions associated with a cardioid," Afrika Matematika, vol. 27, no. 5-6, pp. 923-939, 2016.

[9] S. Kumar and V. Ravichandran, "A subclass of starlike functions associated with a rational function," Southeast Asian Bulletin of Mathematics, vol. 40, no. 2, pp. 199-212, 2016.

[10] K. Bano and M. Raza, "Starlike functions associated with cosine function," Bulletin of Iranian Mathematical Society, 2020.

[11] A. Alotaibi, M. Arif, M. A. Alghamdi, and S. Hussain, "Starlikness associated with cosine hyperbolic function," Mathematics, vol. 8, no. 7, article 1118, 2020.

[12] S. S. Kumar and K. Arora, "Starlike functions associated with a petal shaped domain,” 2020, https://arxiv.org/abs/2010.10072.

[13] M. Arif, L. Rani, M. Raza, and P. Zaprawa, "Fourth Hankel determinant for the family of functions with bounded turning," Bulletin of Korean Mathematical Sciences, vol. 55, no. 6, pp. 1703-1711, 2018.

[14] M. Darus and R. W. Ibrahim, "Partial sums of analytic functions of bounded turning with applications," Computational \& Applied Mathematics, vol. 29, no. 1, pp. 81-88, 2010.

[15] P. T. Mocanu, "On a subclass of starlike functions with bounded turning," Revue Roumaine de Mathématiques Pures et Appliquées, vol. 55, no. 5, pp. 375-379, 2010.

[16] N. Tuneski, "Convex functions and functions with bounded turning," Tamsui Oxford Journal of Mathematical Sciences, vol. 26, no. 2, pp. 161-172, 2010.
[17] C. Pommerenke, "On the coefficients and Hankel determinants of univalent functions," Journal of the London Mathematical Society, vol. 1, no. 1, pp. 111-122, 1966.

[18] C. Pommerenke, "On the Hankel determinants of univalent functions," Mathematika, vol. 14, no. 1, pp. 108-112, 1967.

[19] W. K. Hayman, "On second Hankel determinant of mean univalent functions," Proceedings of the London Mathematical Society, vol. 3, no. 1, pp. 77-94, 1968.

[20] M. Obradović and N. Tuneski, "Hankel determinants of second and third order for the class $\delta$ of univalent functions," https://arxiv.org/abs/1912.06439.

[21] A. Janteng, S. A. Halim, and M. Darus, "Coefficient inequality for a function whose derivative has a positive real part," Journal of Inequalities in Pure and Applied Mathematics, vol. 7, no. 2, pp. 1-5, 2006.

[22] A. Janteng, S. A. Halim, and M. Darus, "Hankel determinant for starlike and convex functions," International Journal of Mathematical Analysis, vol. 1, no. 13, pp. 619-625, 2007.

[23] D. Răducanu and P. Zaprawa, "Second Hankel determinant for close-to-convex functions," Comptes Rendus Mathematique, vol. 355, no. 10, pp. 1063-1071, 2017.

[24] D. V. Krishna and T. RamReddy, "Second Hankel determinant for the class of Bazilevic functions," Studia Universitatis BabeșBolyai Mathematica, vol. 60, no. 3, pp. 413-420, 2015.

[25] Ş. Altınkaya and S. Yalçın, "Upper bound of second Hankel determinant for bi-Bazilevič functions," Mediterranean Journal of Mathematics, vol. 13, no. 6, pp. 4081-4090, 2016.

[26] D. Bansal, "Upper bound of second Hankel determinant for a new class of analytic functions," Applied Mathematics Letters, vol. 26, no. 1, pp. 103-107, 2013.

[27] M. Çaglar, E. Deniz, and H. M. Srivastava, "Second Hankel determinant for certain subclasses of bi-univalent functions," Turkish Journal of Mathematics, vol. 41, no. 3, pp. 694-706, 2017.

[28] S. K. Lee, V. Ravichandran, and S. Supramaniam, "Bounds for the second Hankel determinant of certain univalent functions," Journal of Inequalities and Applications, vol. 2013, no. 1, Article ID 281, 2013.

[29] M. S. Liu, J. F. Xu, and M. Yang, "Upper bound of second Hankel determinant for certain subclasses of analytic functions," Abstract and Applied Analysis, vol. 2014, Article ID 603180, 10 pages, 2014.

[30] K. O. Babalola, "On $H_{3}(1)$ Hankel determinant for some classes of univalent functions," Inequality Theory and Applications, vol. 6, pp. 1-7, 2010.

[31] Ş. Altinkaya and S. Yalçin, "Third Hankel determinant for Bazilevič functions," Advances in Mathematics, vol. 5, no. 2, pp. 91-96, 2016.

[32] D. Bansal, S. Maharana, and J. K. Prajapat, "Third order Hankel determinant for certain univalent functions," Journal of Korean Mathematical Society, vol. 52, no. 6, pp. 1139-1148, 2015.

[33] D. V. Krishna, B. Venkateswarlu, and T. RamReddy, “Third Hankel determinant for bounded turning functions of order alpha," Journal of the Nigerian Mathematical Society, vol. 34, no. 2, pp. 121-127, 2015.

[34] M. Raza and S. N. Malik, "Upper bound of the third Hankel determinant for a class of analytic functions related with lemniscate of Bernoulli," Journal Inequalities and Applications, vol. 2013, no. 1, article 412, 2013. 
[35] G. Shanmugam, B. A. Stephen, and K. O. Babalola, "Third Hankel determinant for $\alpha$-starlike functions," Gulf Journal of Mathematics, vol. 2, no. 2, pp. 107-113, 2014.

[36] P. Zaprawa, "Third Hankel determinants for subclasses of univalent functions," Mediterranean Journal of Mathematics, vol. 14, no. 1, article 19, 2017.

[37] O. S. Kwon, A. Lecko, and Y. J. Sim, "The bound of the Hankel determinant of the third kind for starlike functions," Bulletin of the Malaysian Mathematical Sciences Society, vol. 42, no. 2, pp. 767-780, 2019.

[38] B. Kowalczyk, A. Lecko, and Y. J. Sim, "The sharp bound of the Hankel determinant of the third kind for convex functions," Bulletin of the Australian Mathematical Society, vol. 97, no. 3, pp. 435-445, 2018.

[39] A. Lecko, Y. J. Sim, and B. Śmiarowska, "The sharp bound of the Hankel determinant of the third kind for starlike functions of order 1/2," Complex Analysis and Operator Theory, vol. 13, no. 5, pp. 2231-2238, 2019.

[40] S. Mahmood, H. M. Srivastava, N. Khan, Q. Z. Ahmad, B. Khan, and I. Ali, "Upper bound of the third Hankel determinant for a subclass of $q$-starlike functions," Symmetry, vol. 11, no. 3, article 347, 2019.

[41] H. M. Srivastava, Ş. Altınkaya, and S. Yalçın, "Hankel determinant for a subclass of bi-univalent functions defined by using a symmetric q-derivative operator," Filomat, vol. 32, no. 2, pp. 503-516, 2018.

[42] M. Shafiq, H. M. Srivastava, N. Khan, Q. Z. Ahmad, M. Darus, and S. Kiran, "An upper bound of the third Hankel determinant for a subclass of $q$-starlike functions associated with $k$-Fibonacci numbers," Symmetry, vol. 12, no. 6, article 1043, 2020.

[43] C. Pommerenke, Univalent Function, Vanderhoeck \& Ruprecht, Göttingen, Germany, 1975.

[44] R. J. Libera and E. J. Złotkiewicz, "Early coefficients of the inverse of a regular convex function," Proceedings of the American Mathematical Society, vol. 85, no. 2, pp. 225-230, 1982.

[45] O. S. Kwon, A. Lecko, and Y. J. Sim, "On the fourth coefficient of functions in the Carathéodory class," Computational Methods and Function Theory, vol. 18, no. 2, pp. 307-314, 2018.

[46] F. Keough and E. Merkes, "A coefficient inequality for certain classes of analytic functions," Proceedings of the American Mathematical Society, vol. 20, no. 1, pp. 8-12, 1969.

[47] M. Arif, M. Raza, H. Tang, S. Hussain, and H. Khan, "Hankel determinant of order three for familiar subsets of analytic functions related with sine function," Open Mathematics, vol. 17, no. 1, pp. 1615-1630, 2019.

[48] L. Shi, I. Ali, M. Arif, N. E. Cho, S. Hussain, and H. Khan, "A study of third Hankel determinant problem for certain subfamilies of analytic functions involving cardioid domain," Mathematics, vol. 7, no. 5, article 418, 2019.

[49] M. Arif, S. Umar, M. Raza, T. Bulboaca, M. U. Farooq, and H. Khan, "On fourth Hankel determinant for functions associated with Bernoulli's lemniscate," Hacettepe Journal of Mathematics and Statistics, vol. 49, no. 5, pp. 1777-1780, 2020.

[50] R. J. Libera and E. J. Zlotkiewicz, "Coefficient bounds for the inverse of a function with derivative in $\mathscr{P}$," Proceedings of the American Mathematical Society, vol. 87, no. 2, pp. 251257, 1983. 